\title{
Motivación y Calidad de vida laboral en el personal asistencial del Hospital Hugo Pesce Pescetto, 2020
}

\author{
Liliana Salazar Marcatoma \\ lisama28_291@ hotmail.com \\ https://orcid.org/0000-0003-1441-7782 \\ Medico gineco obstetra del Hospital Hugo Pesce Pescetto
}

\section{RESUMEN}

El objetivo del estudio fue determinar la relación entre motivación y la calidad de vida laboral en el personal asistencial del Hospital Hugo Pesce Pescetto. El diseño del estudio fue no experimental, con enfoque cuantitativo y nivel correlacional, la población de estudio fue de 440 trabajadores asistenciales, seleccionándose una muestra de 206 mediante un muestreo probabilístico aleatorio estratificado. Los instrumentos utilizados fueron: para medir motivación laboral la escala basada en la teoría de autodeterminación de Lohmann et ál., (2017) la misma que mide la composición de la motivación en el personal de salud y para medir calidad de vida laboral se utilizó la escala de CVL-HP de Hernández-Vicente et ál., (2017).Los resultados que se obtuvieron indican que el personal asistencial cuenta con un nivel de motivación laboral alta (87\%), estando la motivación intrínseca y extrínseca en un $88 \%$ y $85 \%$ respectivamente. Por otra parte, la calidad de vida laboral fue predominantemente regular (82\%) y sólo en un $17 \%$ fue buena. Mediante el análisis inferencial se concluyó la relación lineal estadísticamente significativa y moderada entre la motivación y calidad de vida laboral del personal asistencial del Hospital Hugo Pesce Pescetto, 2020 (Rho Spearman = 0.41, $p=0,000$ ).

Palabras Clave: Motivación, Calidad de Vida laboral, personal de salud 


\title{
Motivation and Quality of work life in the care staff of the Hospital Hugo Pesce Pescetto,2020
}

\begin{abstract}
The objective of the study was to determine the relationship between motivation and quality of work life of healthcare personnel of the Hugo Pesce Pescetto Hospital. The study design was non-experimental, with quantitative approach and correlational level, the study population of 440 healthcare workers, selecting a sample of 206 through a stratified random probability sampling. The instruments used were: to measure work motivation, the scale based on the self-determination theory of Lohmann et ál., (2017), the same that measures the composition of motivation in health personnel and to measure quality of work life, the scale de CVL-HP de Hernández-Vicente et ál., (2017). The results obtained indicate that health care personnel have a high level of work motivation (87\%), with intrinsic and extrinsic motivation being $88 \%$ and $85 \%$ respectively. On the other hand, the quality of working life was predominantly fair (82\%) and was only good in $17 \%$. Through the inferential analysis, the statistically significant and moderate linear relationship between motivation and quality of work life of the Hugo Pesce Pescetto Hospital, 2020 (Rho Spearman $=0.41, p=0.000$ ) was concluded.
\end{abstract}

Key words: Motivation, Quality of work life, health personnel

Artículo recibido: 05 de Mayo 2021 Aceptado para publicación: 20 de Junio 2021 Correspondencia: lisama28_291@ hotmail.com Conflictos de Interés: Ninguna que declarar 


\section{INTRODUCCIÓN}

La investigación se realizó en el Hospital Hugo Pesce Pescetto ubicado en el distrito de Andahuaylas del departamento de Apurímac, este nosocomio es la institución referencial de una población de 210355 habitantes de las provincias de Andahuaylas, Chincheros y algunas zonas de Cuso y Ayacucho (ASISHO, 2019). Los recursos humanos asistenciales son 470 trabajadores entre médicos, licenciados en enfermería, obstetras, técnicos y profesionales de apoyo; los mismos que son limitados para atender la desbordante demanda existente (CAP-P HSRA, 2018).

El Hospital elegido para el estudio ha ido mejorando parcialmente su capacidad resolutiva por el crecimiento progresivo en número de los recursos humanos, por la adquisición de parte del equipamiento necesario y medicamentos esenciales y estratégicos; sin embargo, no ha avanzado de manera apropiada en la administración o gestión del recurso humano tal es así que no se cuenta con la información de los niveles de motivación en el personal asistencial que como Judson, Volpp y Detsky (2015) lo refieren es el componente clave de la atención medica de calidad.

La motivación en los trabajadores de la salud no ha sido abordada ni mucho menos medida por las entidades destinadas para tal fin a nivel nacional, pese a existir reportes en torno a los recursos humanos en salud de los años 2017, 2018 y 2019 no se mencionan los tópicos que son de relevancia para nuestro estudio, tal es así que sólo encontramos durante nuestra revisión la publicación realizada por Arroyo, Hartz y Lau (2011) en donde menciona que en los hospitales del MINSA existe un escaso nivel de desarrollo de las condiciones de trabajo y salud del personal que labora en éstos, si bien es cierto que ha habido muchas reformas hasta el momento sin embargo no se ha avanzado en mejorar sustancialmente las condiciones de trabajo en general del personal de salud. El Hospital Hugo Pesce Pescetto no es ajeno a esta realidad.

Lo mencionado líneas arriba podría deberse a que no se proporcionan motivadores a los trabajadores, no se brinda seguridad laboral, las condiciones de trabajo no son apropiadas; también se podría explicar porque no hay un enriquecimiento de la gestión del recurso humano; en resumen, no se brinda una calidad de vida laboral o en el trabajo apropiada a los trabajadores asistenciales, lo vertido coincide con lo sustentado por Ibrahim y Sunday (2020). 
De seguir desconociendo los niveles y sobre todo la composición de motivación, así como los niveles de la calidad de vida en el trabajo del personal asistencial del Hospital Hugo Pesce Pescetto, no se podrá revertir una situación no deseable en la composición de la motivación del personal y los niveles malos o regulares de la calidad de vida laboral, por lo tanto, no se mejorará la calidad de atención al usuario externo.

Por lo expuesto radica la necesidad imperativa de conocer acerca de la motivación laboral que presentan los trabajadores asistenciales del Hospital Hugo Pesce Pescetto y como se relaciona con la calidad de vida en el trabajo.

Dada la gran variedad de teorías existentes sobre motivación humana, no es posible encontrar una definición única del término. Como punto de partida nos puede servir la ofrecida por Ryan y Deci (2000a), quienes interpretan "la motivación como un impulso para actuar. Ese impulso, nacido unas veces del interior del individuo y, otras, generado por causas externas a él, [...]" p.68. En tanto para Robbins y Coulter (2014) "La motivación son los procesos que inciden en la energía, dirección y persistencia del esfuerzo que realiza una persona para alcanzar un objetivo" (p.506). Por último según Nguyen (2020) "la motivación en un contexto laboral se puede definir como el grado de voluntad del individuo para ejercer y mantener un esfuerzo hacia las metas organizacionales" (p.1).

La teoría de la Autoderminación al abordar los conceptos de motivadores intrínsecos y extrínsecos, tiene un enfoque más explicativo del comportamiento humano siendo mucho más realista para ser aplicado a un entorno de los profesionales de la salud a comparación del resto de teorías que están orientadas más para entornos generales, lo mencionado coincide con lo sustentado por de Studer y Knecht (2016). Por lo vertido se decidió trabajar con la teoría de la de la Autoderminación en el estudio

La teoría de la Autodeterminación (SDT) enlaza una metateoría para encuadrar estudios motivacionales, es una teoría consecuente que define orígenes intrínsecos y extrínsecos variados de motivación, y una reseña de los roles respectivos de la motivación intrínseca y los tipos de motivación extrínseca en el desarrollo social y cognitivo y en las divergencias individuales (Ryan y Deci, 2020). La motivación intrínseca lleva a las personas a actuar exclusivamente para satisfacer su deseo de dominio o curiosidad, todas las demás acciones están motivadas extrínsecamente, es decir impulsadas por valores sociales. Las acciones motivadas extrínsecamente pueden volverse auto determinadas a 
medida que los valores se integran e internalizan. Las motivaciones intrínsecas e internalizadas son promovidas por sentimientos de competencia, autonomía y afinidad (Cook y Artino, 2016). Quizás lo más importante de la propuesta de la SDT también se focaliza en cómo los factores de índole social y cultural facilitan o socavan (debilitan) el sentido de iniciativa y voluntad de las personas, además de su calidad de su desempeño y bienestar (Ryan y Deci, 2020).

Las dimensiones de la motivación, según Ryan y Deci (2000a), es en primer lugar la MI que se define como el compromiso con una actividad de forma libre y plena, en tanto la segunda dimensión que es la motivación extrínseca (ME) residiría en la realización de una actividad con la finalidad de recibir un incentivo externo. La primera clase de ME es la regulación externa, en la que la motivación aparece de cara a la obtención de un premio o la evitación de un castigo. En segundo lugar, se tiene a la regulación introyectada, que es un tipo de motivación controlada, una forma de motivación que se genera fuera de la voluntad del individuo, surge frente a sensaciones de culpa o vergüenza causada por el entorno social que se interiorizan en forma parcial. La regulación Identificada (Ryan y Deci, 2000b) en tanto es el tipo de ME que se asocia a un locus interno de causalidad y que puede ser considerada como una forma autónoma de motivación. Por último, la regulación integrada (Ryan a Deci, 2000b), que supone la forma más autónoma de ME y funciona de forma parecida a la motivación intrínseca (Botella y Ramos, 2019).

La importancia de medir la variable motivación se sustenta en: Según Porter, Reisenmy y Fields (2016) para lograr un mejor desempeño de las personas en una organización, se debe lograr y retener una combinación de la motivación y potencial. Está claro que la motivación personal entre los miembros individuales de una organización juega un papel crucial en la determinación del entorno del trabajo general y desempeño de una organización. Legarde, Huicho y Papanicolas (2019) en su revisión encontraron que la baja calidad de atención y los errores médicos ocurren con mayor frecuencia cuando los proveedores están desmotivados, lo que puede verse impulsado por condiciones de trabajo inadecuadas. Se sabe que la motivación intrínseca es un poderoso impulsor de los comportamientos de los proveedores de salud. Tal es así que hay evidencia de que los proveedores intrínsecamente motivados muestran comportamientos o actitudes deseables hacia los pacientes; se ha encontrado que los médicos más generosos brindan una mejor calidad de atención a los pacientes; por lo tanto, si la motivación intrínseca es un rasgo 
individual innato, los responsables políticos deberían intentar seleccionar más personas que muestren este tipo correcto de motivación.

La importancia de abordar la motivación extrínseca radica en que éste tipo de motivación según lo sustenta diferente formuladores de políticas, los mismos que afirman que los motivadores externos y específicamente los económicos son usados para influenciar en el accionar o la conducta del personal de la salud (Legarde et ál., 2019), éstos según Mendelson et ál., (2017) son usados en entornos donde los salarios son bajos, utilizándose para tal efecto esquemas de pago por desempeño con el objetivo de aumentar la remuneración y proporcionar incentivos para mejorar el desempeño de los trabajadores. Según Berdud et ál., (2016) las evidencias de estos esquemas de pago demuestran que no hay impacto positivo y sustancial en la calidad de atención y más al contrario es contraproducente para las personas quienes son impulsadas por motivadores intrínsecos. Con respecto a la definición de calidad de vida laboral tenemos:

"La calidad de vida laboral es parte de la calidad de vida general que está influenciada por el trabajo. Es más que solo satisfacción laboral o felicidad laboral, sino el contexto más amplio en el que un empleado evaluaría su entorno de trabajo" (Varghese y Jayan, 2013b, p.91).

Según Chiavenato (2009) "el termino de calidad de vida laboral o en el trabajo (CVT) fue acuñado por Louis Davis en la década de 1970, cuando desarrollaba un proyecto sobre el diseño de puestos. Según él, el concepto se refiere a una preocupación por el bienestar general y la salud de los colaboradores cuando desempeñan sus actividades [...]. Hoy en día, el concepto de calidad de vida en el trabajo incluye tanto aspecto físicos y ambientales, como los aspectos psicológicos de los trabajadores en su centro de trabajo" (p.492).

Para Hernández-Vicente et ál., (2017) lo más valioso de una organización o empresa para el logro de los objetivos lo constituye el capital humano. Así mismo manifiesta que la CVT engloba todos los esfuerzos para acrecentar la mejora del bienestar y productividad de los trabajadores en su medio laboral. Definen las dimensiones de la CVT que validaron en su instrumento en Tlaxcala para ser empleado en trabajadores de la salud de hospitales públicos de la siguiente manera: 
Dimension1: Bienestar Individual, está en relación a la calidad de vida que percibe el trabajador, en otras palabras, cómo se logra sentir en su entorno laboral. También se puede decir que viene a ser el grado de placer que experimentan los trabajadores cuando realizan sus actividades o labores, en suma, es el estado afectivo en el que se encontrará en determinado momento un trabajador en su organización (Hernández-Vicente et ál.,2017).

Dimensión 2: Condiciones y Medio ambiente de trabajo, en ésta se incluyen una gran gama tanto de actividades como de políticas instauradas en los lugares o ambientes de trabajo con la finalidad de ayudar o apoyar tanto a los trabajadores como empleadores para incrementar o controlar su salud o mejorarla, de ésta manera se favorecerá la competitividad y productividad de las organizaciones o empresas (Hernández-Vicente $e t$ ál.,2017).

Dimensión 3: Organización, está en relación a la estructuración de la relación que se deben dar o existir entre las actividades o funciones del activo primordial o elemento humano de la organización, con la finalidad de que se logre una máxima eficiencia dentro de los objetivos y planes. Para ser más explícitos, los factores inmersos en esta dimensión a citarse están en relación a, los niveles jerárquicos, las reglas existentes, el tipo de conducción de la institución o liderazgo existente, la estructura de la organización, los diseños de empleo, las rotaciones (en relación a estabilidad laboral), el aprovechamiento de la capacidad de los empleados en la toma de decisiones, entre otras (HernándezVicente et ál.,2017).

Dimensión 4: Bienestar logrado a través del trabajo, engloba el estado de satisfacción de todas o parte de las necesidades, las mismas que están en relación con la manera de vivir, incluye también el disfrute de riquezas y bienes que se logran gracias a la realización de la actividad laboral (Hernández-Vicente et ál.,2017).

La importancia de abordar la calidad de vida laboral se sustenta en que, al mejorar las condiciones laborales de los empleados, como su seguridad y protección, es fácil motivar a los colaboradores a trabajar para conseguir las metas organizacionales; tal es así que existe una clara correlación entre la teoría motivacional y la mejora permanente o continua de las condiciones de trabajo (Galli, 2020). De igual manera opina Healy (2016) quien manifiesta que una organización tiende a ser psicológicamente más satisfecho y motivado para cumplir con las tareas de manera efectiva cuando la organización proporciona el equipo y las condiciones de trabajo adecuadas. Para Abbsi et ál.,(2019) la 
calidad de vida laboral es un constructo vital en la vida de los empleados, lo que puede confirmar la eficiencia de la organización y satisfacción laboral. Kumar, Bhat y Ryali (2018) manifiestan que para hacer frente a los desafíos en el sistema de prestación de los servicios de salud y garantizar la calidad de la atención brindada y la satisfacción del usuario con la atención recibida, es importante conocer que tan satisfechos están los trabajadores de la salud con su calidad de vida en el trabajo, por ende las organizaciones que se centran en garantizar la mejor calidad de trabajo a sus trabajadores son las que tienen mejor rendimiento y el logro de sus objetivos. Para Castro et ál., (2018) es crucial encontrar o identificar los aspectos que son parte de la calidad de vida en el trabajo pues de ello dependerá la satisfacción de las innumerables necesidades de los colaboradores, los mismos que requieren un reconocimiento, equilibrio entre familia y empleo, estabilidad en el trabajo, seguridad, una remuneración equitativa y no podía faltar la motivación.

\section{MATERIALES Y MÉTODOS}

La investigación fue de tipo básica, con enfoque cuantitativo el diseño fue no experimental y de nivel correlacional, que buscó evaluar la relación entre las variables de motivación y calidad de vida laboral (CVT), así como las dimensiones de la motivación y la CVT en el personal de salud.

La población del estudio estuvo constituida por 440 trabajadores asistenciales del Hospital Hugo Pesce Pescetto, los grupos ocupacionales fueron: 57 médicos, 119 enfermeros (as), 48 obstetras, 174 técnicos (as), 42 profesionales de apoyo (Odontólogos, asistentas sociales, nutricionistas, químicos farmacéuticos, biólogos, psicólogos y tecnólogos médicos). Se excluyó al personal asistencial con tiempo de servicio menor a 3 meses, personal asistencial que se encontró realizando labor administrativa, los que se encontraron en área exclusiva de COVID y aquellos con aislamiento prolongado (mayor o igual a 02 meses) por motivos de pandemia. El muestreo fue el probabilístico de tipo aleatorio estratificado resultando una muestra de 206 trabajadores asistenciales, la misma que se calculó con un nivel de confianza de $95 \%$ y un margen de error de $5 \%$. Respetando la proporción de los grupos ocupacionales la muestra quedó constituida de: 27 médicos, 56 enfermeros (as), 22 obstetras, 81 técnicos (as) y 20 profesionales de apoyo.

La técnica fue la encuesta y el instrumento el cuestionario autoadministrado (la misma que contenía la escala de medición); La motivación fue medida mediante la escala basada 
en la Teoría de la Autoderminación de Lohmann et ál., (2017). Este instrumento comprende de 15 ítems valorados sobre una escala visual de Likert de 0 a 10, donde $0=$ No es importante para mí en absoluto y $10=$ Extremadamente importante para mí. Valora dos dimensiones: La motivación intrínseca con 3 ítems (1 al 3) y la motivación extrínseca con 12 ítems (4 al 15), ésta última con 04 indicadores la Regulación integrada/identificada (IDEN), Regulación introyectada (INTRO), Regulación externa social (EXT-S) y la Regulación externa económica (EXT-E). La escala fue adaptada por Lohman et ál., para ser aplicado a trabajadores de la salud y tiene una consistencia interna muy alta (alfa de Cronbach $=0.996$ ). La escala en mención está en idioma extranjero (Inglés y francés) la misma que fue traducida y validad por juicio de 03 expertos.

La evaluación de la variable calidad de vida laboral se realizó usando la escala CVP-HP de Hernández-Vicente et ál., (2017). Esta escala fue diseñada para ser aplicada a personal de hospitales públicos y consta de 55 ítems valorado sobre una escala de Likert de 1 a 3 donde: 1 es malo, 2 regular y 3 bueno. Valora 04 dimensiones: Bienestar Individual, las condiciones y Medio Ambiente, Organización y Bienestar logrado a través del trabajo. La CVP - HP se encuentra en castellano y tiene una consistencia interna muy alta (alfa de cronbach de 0.921).

El análisis estadístico consistió en primer lugar en la estadística descriptiva para las dos variables. Se evaluó la Motivación y la calidad de vida laboral en sus dimensiones por separado mediante sus frecuencias absolutas y porcentuales. La relación entre las dos variables, así como las dimensiones de la motivación con la calidad de vida laboral se evaluó mediante la Rho de Spearman. El nivel de alfa que se determinó previo al análisis fue de 0.05. El análisis se realizó con el programa estadístico SPSS versión 25.0 El estudio materia de presentación se realizó bajo los marcos de la integridad científica y el respeto estricto de los derechos humanos; dando estricto cumplimiento al código de ética en investigación. 


\section{RESULTADOS}

\section{Motivación laboral}

Tabla 1: Nivel de motivación laboral del personal asistencial del Hospital Hugo Pesce

Pescetto, 2020 según dimensiones/Indicadores.

\begin{tabular}{lcccccc}
\hline \multicolumn{1}{c}{ Variable y Dimensiones de la } & \multicolumn{3}{c}{ Bajo } & \multicolumn{2}{c}{ Medio } & \multicolumn{2}{c}{ Alto } \\
\cline { 2 - 8 } \multicolumn{1}{c}{ Motivación laboral/ Indicadores } & fr & \% & fr & $\%$ & fr & $\%$ \\
\hline Motivación laboral & 0 & 0 & 26 & 13 & 180 & 87 \\
Dimensión Intrínseca (MI) & 2 & 1 & 22 & 11 & 182 & 88 \\
Dimensión Extrínseca (ME) & 1 & 1 & 29 & 14 & 176 & 85 \\
Regulación Integrada/Identificada & 1 & 0 & 14 & 7 & 191 & 93 \\
(IDEN) & & & & & & \\
Regulación Introyectada (INTRO) & 3 & 2 & 10 & 5 & 193 & 93 \\
Regulación externa social (EXT-S) & 10 & 5 & 34 & 16 & 162 & 79 \\
Regulación externa económica (EXT-E) & 13 & 6 & 44 & 22 & 149 & 72 \\
\hline
\end{tabular}

Fuente: Elaboración propia-Base de datos.

En la tabla 1 se observa la variable motivación en el entorno laboral del personal asistencial del Hospital Hugo Pesce Pescetto en donde el $87 \%$ obtuvieron un nivel o intensidad de motivación laboral alta, mientras que un $13 \%$ obtuvieron un nivel de motivación laboral media; así mismo se desprende que la motivación intrínseca presentó predominantemente un nivel alto en un $88 \%$, por su parte la motivación extrínseca tuvo un nivel bajo en sólo un $1 \%$ estando predominantemente alto. Si analizamos esta última dimensión por indicadores o tipos de regulación tenemos que la IDEN y la INTRO presentaron niveles sustancialmente altos ambos con 93\%, en tanto que las regulaciones EXT-S Y EXT-E presentaron niveles bajos que son deseables en sólo un 5\% y $6 \%$ respectivamente.

Tabla 2: Nivel de calidad de vida laboral del personal asistencial del Hospital Hugo Pesce Pescetto, 2020 según dimensiones.

\begin{tabular}{lccccccc}
\hline Variable y dimensiones de la calidad de & \multicolumn{2}{c}{ Malo } & \multicolumn{2}{c}{ Regular } & \multicolumn{2}{c}{ Bueno } \\
\cline { 2 - 7 } \multicolumn{1}{c}{ vida laboral } & fr & $\mathbf{\%}$ & Fr & $\%$ & fr & $\%$ \\
\hline Calidad de Vida laboral & 3 & 1 & 168 & 82 & 35 & 17 \\
Bienestar Individual & 3 & 2 & 149 & 72 & 54 & 26 \\
Condiciones y medio ambiente & 3 & 2 & 187 & 90 & 16 & 8 \\
Organización & 5 & 2 & 156 & 76 & 45 & 22 \\
Bienestar logrado a través del trabajo & 2 & 1 & 121 & 59 & 83 & 40 \\
\hline
\end{tabular}

Fuente: Elaboración Propia-Base de datos 
De la tabla 2 que evalúa la variable calidad de vida laboral de los trabajadores asistenciales del hospital Hugo Pesce Pescetto se desprende que el nivel de calidad de vida en el trabajo fue predominantemente regular con un $82 \%$ mientras que sólo un $17 \%$ tiene una calidad de vida laboral buena, así mismo se desprende que las condiciones y medio ambiente de la calidad de vida laboral fue la dimensión con mayor nivel de presentación regular en un 90\%, seguido de la organización con un $76 \%$ en tanto el bienestar logrado a través del trabajo sólo contó con un 59\% de presentación regular. Cabe recalcar que el bienestar logrado a través del trabajo fue la dimensión con mayor nivel de presentación buena con un $40 \%$ en comparación con el resto de dimensiones.

\section{Contrastación de hipótesis}

Teniendo en consideración que los datos obtenidos provienen de cuestionarios con escala ordinal, se asume el empleo de una prueba no paramétrica para demostrar la hipótesis general y las específicas del estudio, las mismas que se corroboraron mediante la prueba de Kolmokorov-Smirnov, empleándose por ello la prueba no paramétrica de Rho de Spearman y el reporte del programa SPSS 25.

\section{Contrastación de hipótesis general}

Tabla 3: Correlación entre la motivación y calidad de vida laboral del personal asistencial del Hospital Hugo Pesce Pescetto, 2020.

\begin{tabular}{lccc}
\hline & \multicolumn{3}{c}{ Calidad de Vida laboral } \\
\cline { 2 - 4 } & $\mathrm{N}$ & $\begin{array}{c}\text { Coeficiente de } \\
\text { correlación }\end{array}$ & Sig. (bilateral) \\
\hline Rho de Spearman Motivación laboral & 206 &, 413 &, 000 \\
\hline
\end{tabular}

Fuente: Base de datos-Procesamiento con SPSS

En la tabla 3 se observa que $\mathrm{p}<0.05$ por lo que en consecuencia se desestima la hipótesis nula y se colige que existe una relación directa entre la motivación y la calidad de vida laboral en el personal asistencial del Hospital Hugo Pesce Pescetto, 2020. También se infiere que la motivación laboral está relacionada directa y positivamente con la calidad de vida laboral (rho de Spearman $=0.413$ ), siendo el grado de correlación positiva moderada. 


\section{Contrastación de la hipótesis especifica 1}

Tabla 4: Correlación entre la motivación intrínseca y calidad de vida laboral del personal asistencial del Hospital Hugo Pesce Pescetto, 2020.

\begin{tabular}{llccc}
\hline & & \multicolumn{3}{c}{ Calidad de Vida laboral } \\
\cline { 3 - 5 } & N & $\begin{array}{c}\text { Coeficiente de } \\
\text { correlación }\end{array}$ & Sig. (bilateral) \\
\hline Rho de Spearman & $\begin{array}{l}\text { Motivación } \\
\text { Intrínseca }\end{array}$ & 206 &, 301 &, 000 \\
\hline
\end{tabular}

\section{Fuente: Base de datos-Procesamiento con SPSS}

En la tabla 4 se observa que $\mathrm{p}<0.05$ por lo que se desestima la hipótesis nula y se colige que existe relación directa entre la motivación intrínseca y la calidad de vida laboral en el personal asistencial del Hospital Hugo Pesce Pescetto, 2020. También se infiere que la dimensión intrínseca se relaciona directa y positivamente con la calidad de vida laboral (rho de Spearman $=0.301)$. Siendo el grado de correlación positiva débil.

\section{Contrastación de la hipótesis especifica 2}

Tabla 5: Correlación entre la motivación extrínseca y calidad de vida laboral del personal asistencial del Hospital Hugo Pesce Pescetto, 2020.

\begin{tabular}{llccc}
\hline & & \multicolumn{3}{c}{ Calidad de Vida laboral } \\
\cline { 3 - 5 } & N & $\begin{array}{c}\text { Coeficiente de } \\
\text { correlación }\end{array}$ & Sig. (bilateral) \\
\hline Rho de Spearman & Motivación & 206 &, 392 &, 000 \\
& Extrínseca & & & \\
\hline
\end{tabular}

Fuente: Base de datos-Procesamiento con SPSS

En la tabla 5 se observa que $\mathrm{p}<0.05$ por lo que se desestima la hipótesis nula y se colige que existe una relación directa entre la motivación extrínseca y la calidad de vida laboral en el personal asistencial del Hospital Hugo Pesce Pescetto, 2020. También se infiere que la motivación extrínseca está relacionada directa y positivamente con la calidad de vida laboral (rho de Spearman $=0.392$ ). Siendo el grado de correlación positiva débil.

\section{DISCUSION}

Según lo hallado en la investigación, existe relación directa entre la motivación y la calidad de vida laboral en el personal asistencial del Hospital en estudio, por lo que en un principio se identificaron los niveles o intensidad de motivación laboral y su composición 
a través de la escala de medición de motivación laboral de Lohmann et ál., (2017) la misma que está basada en la Teoría de la Autodeterminación. Señalar que la medición y el análisis de la motivación no son sencillos debido a que la motivación no es directamente observable, sin embargo, pese a no ser una experta en psicología, se ha dado nuestro mejor esfuerzo en base a la literatura para brindar las explicaciones basadas en la evidencia existente.

Tras la aplicación de la escala de motivación laboral se determinó la existencia de niveles altos de motivación en el personal asistencial del Hospital Hugo Pesce Pescetto con un $87 \%$ y niveles medios de motivación con sólo un 13\%. Estos resultados generales de motivación laboral deben ser tomados con cautela, pues las respuestas a las preguntas sobre la motivación pueden haberse visto afectados por el sesgo de deseabilidad social, lo cual es muy difícil de controlarlo en un instrumento auto administrado (Borghi et ál., 2018) (Lohmann et ál., 2017). Por ejemplo, los encuestados pueden proporcionar puntuaciones altas en motivadores intrínsecos (elementos relacionados deseables al compromiso o la actitud hacia el trabajo) independientemente de cómo se sientan realmente.

Los resultados generales en motivación laboral hallados en la investigación guardan relación con los reportados por Abate et ál., (2020) quien también en su estudio transversal realizado en Etiopia reporta niveles altos de motivación en los trabajadores sanitarios, siendo relevantes para nuestro estudio el hallazgo de que los trabajadores que tenían una carga de trabajo media eran significativamente los menos motivados en comparación con aquellos con una carga de trabajo ligera; por otro lado el reconocimiento y el apoyo fue significativamente más alto entre los más motivados es decir tener más reconocimientos fue un predictor positivo de satisfacción laboral (indicador de calidad de vida laboral).

Comparando los resultados con el estudio de Soto (2018) estos también guardan relación, pues este último encontró con respecto a los factores motivadores del establecimiento Centro de salud esperanza en un nivel alto $(77.92 \%)$ el cual se corresponde a la motivación intrínseca, de igual modo para los factores higiénicos halló un nivel alto con un $66.23 \%$ el cual se corresponde con nuestro estudio con la motivación extrínseca, las variaciones en los porcentajes pueden ser explicados a que el estudio de Soto (2018) solo se realizó en 77 trabajadores de salud, incluido personal administrativo y de servicio; 
éstos dos últimos grupos ocupacionales con características distintas al personal netamente asistencial, la cual fue incluida exclusivamente en nuestro estudio. De igual modo las variaciones se pueden explicar por el instrumento utilizado fue según la Teoría de Herzberg en relación a la nuestra que fue según la teoría de Autodeterminación.

La escala psicométrica de Lohmann et ál., (2017) utilizada la misma que fue elaborada para mensurar la composición de la motivación laboral, es decir el grado en que la motivación de diferente origen ya sea de dentro o de afuera de una persona contribuye a su motivación general, tal es así que tenemos los resultados según dimensión de la motivación laboral que se obtuvieron en el estudio en donde la motivación intrínseca fue reportada en un nivel alto $88 \%$ del personal asistencial y la motivación extrínseca también reportó niveles similares con un 85\%. Según la Teoría de Ryan y Deci (2020) de los resultados por dimensión podemos indicar que la motivación intrínseca o altruista es aquella que mueve al personal de salud por el disfrute inherente hacia sus actividades y es un atributo deseable para todo personal de salud. La motivación intrínseca es un poderoso impulsor de los comportamientos de los proveedores de la salud; según las evidencias los trabajadores intrínsecamente motivados muestran comportamientos y actitudes deseables hacia los pacientes (Legarde et ál., 2019).

Los resultados de la motivación extrínseca llaman la atención por sus altos niveles, este tipo de motivación que según Ryan y Deci (2000a) es generado por causas externas como, por ejemplo, la remuneración, los incentivos financieros, la reputación, la evitación de castigos, entre otros. Estos deberían estar en niveles bajos para ser considerados deseables en el personal de salud; sin embargo, en el estudio los indicadores o tipos de regulación involucrados en esta dimensión (regulación Introyectada, regulación externa social y económica) se encuentran en niveles altos, al respecto Legarde et ál., (2019) sostiene es su revisión que ninguna cantidad de dinero o incentivo económico mejorará las habilidades clínicas para hacer un diagnóstico correcto o mejorar la calidad de atención si no se combina con una actitud altruista (motivación intrínseca) disposición de medicamentos y equipos esenciales; más al contrario la dotación de motivadores externos socavan la motivación intrínseca, pues las personas se verán impulsadas a trabajar por el incentivo financiero más no por sus pacientes o su trabajo.

Con respecto a los niveles de calidad de vida laboral, lo obtenido indica que el personal asistencial del Hospital Hugo Pesce Pescetto tiene una calidad de vida laboral 
predominantemente regular con un $82 \%$, esto quiere decir que el personal asistencial evalúa en término medio la organización de la institución, la atención de necesidades de capacitación, la satisfacción laboral y de los insumos, las condiciones y medio ambiente así como las demandas y expectativas de los mismos (Hernández-Vicente et ál., 2017). Los resultados en torno a esta variable de calidad de vida laboral pese a la utilización de instrumentos distintos son semejantes a lo hallado en la tesis de Ramírez (2017) quien encontró una calidad de vida profesional regular predominantemente en el servicio de emergencia de un hospital de Lima en un $86.08 \%$, los resultados también son similares a los hallados por Malca (2016) en Lima quien encontró un 85\% de personal que considera la calidad de vida laboral en un nivel medio o regular. Merece mención el trabajo de Ibrahim y Sunday (2020) en Nigeria quien sostiene que las condiciones de trabajo no apropiadas son desafíos de la realización profesional.

Los resultados de Ruiz-Fernández et ál., (2020) en relación a la variable calidad de vida laboral medido con el instrumento PQoL no concuerdan con lo hallado en el estudio, pues ellos encontraron niveles altos de calidad de vida laboral en un $64.1 \%$ del personal de salud de enfermería, estos resultados se pueden explicar porque se trata de un estudio multicéntrico realizado en otra realidad como es España un país desarrollado a comparación del nuestro en donde el sistema de salud tiene muchas limitaciones y es de esperar que el personal de salud reporte niveles regulares con mayor frecuencia. Al respecto Kumar et ál., (2018) sostiene que para enfrentar los desafíos en un sistema de salud y garantizar calidad de atención al usuario externo es necesario conocer primero que tan satisfechos están los trabajadores asistenciales con su calidad de vida en el trabajo, razón por la cual las entidades que se centran en mejorar la calidad de trabajo a sus colaboradores son las que tienen mejor rendimiento y logro de sus objetivos organizacionales.

Con relación a la hipótesis general, los resultados del estudio demostraron la asociación directa entre la motivación y calidad de vida laboral en el personal asistencial del Hospital Hugo Pesce Pescetto con un Rho de Spearman $=0.413$, lo que significa que existe una correlación positiva moderada, éste se asemeja a los resultados obtenidos en el estudio de Moller et ál., (2019) en Estados Unidos, quienes informaron que los médicos más motivados y específicamente con los mayores niveles de motivación autónoma (regulación intrínseca, integrada e identificada) son los que informan tener mejor salud 
ocupacional (indicador de calidad de vida laboral) y específicamente a mayor motivación autónoma se asocia una mejor salud general, menor riesgo para la depresión, disminución del agotamiento, aumento de satisfacción laboral y una menor intención de dejar la práctica actual. También los resultados se apoyan en la revisión de Porter et ál., (2019) quienes indican que la motivación laboral de los miembros individuales de una organización juega un papel crucial en la determinación del entorno del trabajo y desempeño de una organización.

También lo hallado en el estudio se sustenta en lo mencionado por Galli (2020) quien indica que, al mejorar las condiciones laborales de los trabajadores, como su seguridad y protección, es fácil motivar a los empleados de tal manera que se pueda alcanzar las metas organizacionales a razón de ello es que existe una clara correlación con la teoría motivacional y la mejora continua de las condiciones de trabajo. De igual modo los resultados se apoyan en la revisión de Healy (2016) quien sostiene que las organizaciones más satisfechas y motivadas para cumplir con las tareas encomendadas de manera efectiva es cuando la organización le proporciona el equipo y las condiciones de trabajo adecuadas.

El resultado general también se asemeja a la tesis de Ramírez (2017) en Lima, quien determinó también la existencia de correlación positiva moderada entre las variables de calidad de vida del profesional de la salud del servicio de emergencias del Hospital Hipólito Unanue y la motivación laboral con un Rho de Spearman $=0.561$. Lo sostenido por Hasugian et ál., (2020) en Indonesia también se asemejan en parte a nuestro estudio pues hallaron que la motivación fue el $51 \%$ de la varianza de la satisfacción laboral, estando este último en relación a el bienestar logrado a través del trabajo.

Los resultados generales de la investigación difieren de lo hallado por Pebes et ál., (2019) en Ica de igual modo el estudio de Collantes y Arevalo (2017) en Lima quienes no encontraron relación existente entre la motivación y la satisfacción laboral (indicador de calidad de vida en el trabajo) con un $p$ de 0.183 y Rho Spearman de 0.191 , estos resultados se puede explicar porque ambos estudios solo incluyeron muestras muy pequeñas de 24 y 50 trabajadores de la salud respectivamente en relación a la nuestra que fue mucho mayor (206 trabajadores asistenciales); además que solo incluyeron licenciados (as) en enfermería a diferencia del nuestro que incluyo todos los grupos ocupacionales por otra parte los estudios arriba mencionados utilizaron Instrumentos distintos tales como son el 
Diagnostic Survey Hackman y Oldham para medir motivación y el cuestionario del MINSA para medir satisfacción laboral.

En relación a la primera hipótesis especifica se determinó que existe una relación directa entre la motivación intrínseca y la calidad de vida laboral en el personal asistencial del Hospital Hugo Pesce Pescetto con un Rho de Spearman de 0.301 lo que nos indica una correlación positiva débil. Los resultados se asemejan al estudio de Marín y Placencia (2017) quienes en su estudio también determinaron una correlación positiva baja (Rho 0.346) entre la motivación laboral en los factores motivacionales y la satisfacción laboral, siendo este último un indicador de la calidad de vida laboral. Señalar que la motivación laboral en los factores motivacionales es señalada según la teoría de Herzberg lo que se asemeja a la del estudio a la motivación intrínseca que es basado en la Teoría de Autoderminación de Ryan y Deci (2000a). Los resultados hallados también son consistentes a los obtenidos por Ramírez (2017) quien encontró una relación positiva baja entre la calidad de vida laboral y la motivación intrínseca. Lo sostenido por Legarde et ál., (2019) apoya a lo hallado pues sostiene que, aunque las buenas condiciones laborales son una parte importante para brindar una atención de buena calidad, no son suficientes para garantizar que los profesionales de la salud estén motivados.

En relación a la segunda hipótesis especifica se resolvió que existe relación directa entre la motivación extrínseca y la calidad de vida laboral con un Rho de Spearman $=0.392$ indicándonos una correlación positiva débil entre la segunda dimensión de motivación laboral y la variable calidad de vida en el trabajo. Según Castro et ál., (2018) para que los trabajadores cuenten con calidad de vida laboral adecuada se requiere que posean un trabajo motivante y enriquecedor, que además les brinde bienestar y equilibrio, que proporcione desarrollo laboral y sea satisfactorio en un ambiente favorable y que acorde a su trabajo se le retribuya económicamente (motivador extrínseco) por la labor justa desempeñada.

De igual forma los resultados también se apoyan en la revisión de Legarde et ál., (2019) quien sostiene que los economistas, gerentes y formuladores de políticas han visto desde hace mucho tiempo la remuneración (motivador externo) como una palanca obvia para influir en el comportamiento de los proveedores de la salud. En entornos donde los salarios son bajos se utiliza esquemas de pago por desempeño con el objetivo de aumentar la remuneración y proporcionar incentivos para mejorar el desempeño (Mendelson et ál., 
2017). A pesar del entusiasmo por estos esquemas, la evidencia de su impacto en la calidad de la atención es mediocre y puede resultar contraproducente para las personas impulsadas por factores distintos a la remuneración (Berdud et ál.,2016).

El instrumento utilizado incluye los ítems necesarios para medir la regulación externa económica arriba descrita y la regulación externa social es decir la preocupación por la reputación como motivador laboral (Lohmann et ál., 2017), los cuales se encuentran en niveles altos al igual que la regulación externa económica; al respecto tenemos que mencionar que si el personal no tiene las habilidades clínicas para hacer por ejemplo un diagnóstico correcto, ninguna cantidad de dinero u otros incentivos aumentara la calidad técnica de atención (Legarde et ál., 2019) así mismo los estudios de nivel de evidencia IA no han demostrado que los incentivos económicos mejoren los resultados de la salud en ningún entorno tampoco se ha podido establecer ningún dato para determinar que incentivo es el más exitoso (Mendelson, et ál., 2017). Según la teoría de Maslow que establece que las personas buscan primero satisfacer sus necesidades fisiológicas y de seguridad más urgentes (Faizul et ál.,2014); para el caso del estudio al tener altos niveles de regulación externa económica esto se puede explicar porque aún la remuneración es una prioridad para satisfacer las necesidades esenciales del personal asistencial en estudio, por lo que aún no están motivados esencialmente de manera autónoma (regulación intrínseca, integrada e identificada), esto según la teoría de la Autodeterminación de Ryan y Deci (2000a).

De los resultados obtenidos podemos mencionar que el personal asistencial del Hospital Hugo Pesce Pescetto están motivados por una serie de factores, tanto intrínsecos como extrínsecos tal como lo menciona Lateff (2020). Tal es así que esta descrito que las recompensas monetarias y la motivación intrínseca interactúan a veces como complemento (cuando los incentivos afectan positivamente la motivación intrínseca) y como sustitutos (cuando los incentivos afectan negativamente) teniendo en consideración que los trabajadores son intrínsecamente motivados porque obtienen el disfrute del mero hecho de realizar una actividad, u obtienen utilidad de los sentimientos de automotivación en lugar de estar controlados por recompensas o contingencias impuestas externamente (Ryan y Deci, 2000a). Sin embargo, los estudios han probado que las retribuciones extrínsecas pueden desplazar a la motivación intrínseca; tal es así que una gran variedad 
de la literatura a abordado el tema de los trabajadores no motivados económicamente en el contexto de organizaciones públicas y sin fines de lucro (Berdud et ál.,2016).

Si bien la motivación de los trabajadores de la salud en los países en vías de desarrollo se puesto en la palestra y se ha convertido en los últimos años en un tema de debate y creciente interés por parte de los responsables políticos, así como de investigadores, muchos aspectos no se comprenden bien hasta la fecha. De igual forma en torno a la calidad de vida laboral no existe una definición clara y aceptada debido a la gran diversidad de enfoques e intereses de las diferentes organizaciones. Sin embargo, lo que, si podemos inferir en relación a los resultados del estudio y apoyándonos en la revisión de Castro, et ál., (2018) es que un lugar de trabajo equilibrado donde el trabajador posea suficientes recursos para su bienestar individual, contando con un ambiente apropiado y una organización óptima éstos serán cruciales en parte para la motivación de los trabajadores por ende mejorará su calidad de atención el cual redundará en beneficio de la entidad, así como para los usuarios.

\section{RECOMENDACIONES}

Al ministerio de salud se le recomienda para hacer frente a los desafíos en el sistema de prestaciones de salud y garantizar la calidad de atención brindada y la satisfacción del usuario externo con la atención recibida, realizar una encuesta nacional como línea de base para conocer la composición de la motivación laboral y que factores son los verdaderamente determinantes de la calidad de vida laboral de los trabajadores asistenciales, pues esto servirá para la realización de intervenciones en relación al recurso humano que son el activo principal de una organización y que hasta el momento pese a haberse dado una serie de reformas siguen relegados.

A los responsables de las oficinas de recursos humanos, teniendo en consideración que la motivación intrínseca es un rasgo individual innato y deseable para los recursos humanos en salud, entonces los responsables de la contratación de los recursos humanos asistenciales deberían intentar seleccionar más personas que muestren este tipo correcto de motivación con la introducción de una escala de motivación en el proceso de selección de personal o mejor aún se debería introducir programas para identificar a los trabajadores de la salud con rasgos de personalidad y valores indicativos de motivación intrínseca y altruista y que a su vez muestren niveles bajos de motivación extrínseca. 
Al Gobierno disminuir las intervenciones diseñadas para aumentar la motivación extrínseca con incentivos económicos, pues promover una mayor regulación externa con incentivos de este tipo es ineficaz para mejor la calidad de atención. De hecho, los datos sugieren que los incentivos extrínsecos pueden socavar la motivación intrínseca de los trabajadores de la salud. Se debe de buscar por lo tanto otras formas de incentivar al personal de salud para optimizar la calidad de su atención, no necesariamente económicas pero que impulsen a los proveedores de la salud a desempeñarse mejor.

\section{REFERENCIAS}

Arroyo, J., Hartz, J., y Lau, M. (2011). Recursos Humanos en salud al 2011. Lima-Perú. http://bvs.minsa.gob.pe/local/minsa/1736.pdf

Abate Adillo, M., Mulissa, Z., Bitewulign Desta, B., Kiflie Alemayehu, A., Biadgo Kefale, A., Woldesenbet, D., . . . Quaife, M. (2020). Key factors influencing motivation among health extension workers and health care professionals in four regions of Ethiopia: a cross-sectional study. Research Square, 1-24. doi:10.21203/rs.3.rs-15944/v1

Abbsi, M., Farhang Dehghan, S., Fallah Madvari, R., Mehri, A., Hossein Ebrahimi, M., Poursadeghyan, M., . . Ghaljahi, M. (2019). Interactive Effect of Background Variables and Workload Parameters on the Quality of Life among Nurses Working in Highly Complex Hospital Units: A Cross-sectional Study. Journal of Clinical and Diagnostic Research, 13(1), 8-13. doi:10.7860/JCDR/2019/37929.12482

ASISHO. (2018). Analisis Situacional de Salud Hospital Sub Regional de Andahuaylas. Analisis situacional, Hospital Subregional de Andahuaylas, Apurimac, Andahuaylas.

Berdud, M., Cabasés, J., \& Nieto, J. (2016). Incentives and intrinsic motivation in healthcare. Gac Sanit, 30(6), 408-414. http://scielo.isciii.es/pdf/gs/v30n6/02139111-gs-30-06-00408.pdf

Borghi, J., Lohmann, J., Dale, E., Meheus, F., Goudge, J., Oboirien, K., \& Kuwawenaruwa, A. (2018). How to do (or not to do)... Measuring health worker motivation in surveys in low- and middle-income countries. Health policy and planning, 33(2), 192-203. https://doi.org/10.1093/heapol/czx153. 
Botella Nicolas, A., y Ramos Ramos, P. (2019). La teroría de la autodeterminacion: Un marco motivacional para el aprendisaje basado en proyectos. Contextos Educativos, O(24), 253-269. https://doi.org/10.18172/con.3576

CAP-P HSRA. (2018). Hospital Subregional de Andahuylas, Portal Institucional. http://www.hospitalandahuaylas.gob.pe/images/institucion/CAP_2018_RRHH.p df

Castro, P., Cruz, E., Hernández, J., Vargas, R., Luis, K., Gatica, L., \& Tepal, I. (2018). Una Perspectiva de la Calidad de Vida Laboral. Revista Iberoamericana de Ciencias, 5(6), 118-128. http://www.reibci.org/publicados/2018/dic/3200115.pdf

Chiavenato, I. (2009). Gestion del Talento Humano (Tercera Edicion ed.). Mexico. https://cucjonline.com/biblioteca/files/original/338def00df60b66a032da556f56c 28c6.pdf

Collantes Zapata, J., y Arévalo Ipanaque, J. (2017). Motivation and labor satisfaction of the nurse in a Lima-Peru hospital. Rev. Cienc. Arte Enferm., 2(2), 19-24. doi:10.24314/rcae.2017.v2n2.04

Cook, D. A., \& Artino, A. R., Jr (2016). Motivation to learn: an overview of contemporary theories. Medical education, 50(10), 997-1014. https://doi.org/10.1111/medu.13074

Faizul Haque, M., Aminul Haque, M., \& Islam, S. (2014). Motivational Theories-A Critical Analysis. Research Gate, 8(1), 61-68. https://www.researchgate.net/publication/306255973

Galli, B. (2020). Impact and Role of Motivation Theories in Continuous Improvement Environments A Reflection of Literature. International Journal of Service Science, Management, Engineering, and Technology, 11(1), 1-13. doi:10.4018/IJSSMET.2020010101

Hasugian, A., Ilyas, J., Besral, y Bachtiar. (Enero de 2020). Determinants that Influence Relationship between Motivation and Job Satisfaction of Health Workers at Primary Health Care in Indonesia. Indian Journal Of Public Health Reseatch \& Development, 11(1), 369-373. doi:10.37506/v11/i1/2020/ijphrd/193962

Healy, K. (2016). A Theory of Human Motivation by Abraham H. Maslow (1942)reflection. The British Journal of Psychiatry, 208(4), 313-313. doi:10.1192/bjp.bp.115.179622 
Hernandez-Vicente, I., Lumbreras Guzman, M., Mendez Hernandez, P., Rojas Lima, E., Cervantes Rodriguez, M., y Juarez Flores, C. (2017). Validación de una escala para medir la calidad de vida laboral en hospitales públicos de Tlaxcala. Salud pública de méxico, 59(2), 183-192. http://dx.doi.org/10.21149/7758

Ibrahim Ayandare, A., \& Sunday Olanrewaju, P. (2020). Effects of Work Motivation and Self-Esteem on Career Fulfillment Among Health Information Management Personnel in Federal Teaching Hospitals in Southern Nigeria. Jornal of Human Resource Management, 8(2), 85-95. doi: 10.11648/j.jhrm.201200802.16

Judson, T., Volpp, K., \& Detsky, A. (2015). Harnessing the Right Combination of Extrinsic and Intrinsic Motivation to Change Physician Behavior. JAMA, 34(21), 2233-2234. doi: $10.1001 /$ jama.2015.15015

Kumar, A., Bhat, P. S., \& Ryali, S. (2018). Study of quality of life among health workers and psychosocial factors influencing it. Industrial psychiatry journal, 27(1), 96102. https://doi.org/10.4103/ipj.ipj_41_18.

Lateef, F. (9 de Agosto de 2020). Face to face with coronavirus disease 19: Maintaining motivation, psychological safety, and wellness. J Emerg Trauma Shoc, 13(2), 116-123. Obtenido de http://www.onlinejets.org/text.asp?2020/13/2/116/286230

Legarde, M., Huicho, L., \& Papanicolas, I. (2019). Motivating provision of high quality care: it is not all about the money. BMJ, 366(15210), 1-5. https://doi.org/10.1136/bmj.15210

Lohmann, J., Souares, A., Tiendrebéogo, J. et al. (2017). Measuring health workers' motivation composition: validation of a scale based on Self-Determination Theory in Burkina Faso. Hum Resour Health, 15 (33), 1-12. https://doi.org/10.1186/s12960-017-0208-1

Marin Samanez, H., y Placencia Medina, M. (2017). Motivacion y Satisfaccion laboral del personal de una organizacion de salud del sector privado. Horiz Med, 17(4), 42-52. doi:10.24265/horizmed.2017.v17n4.08

Mendelson, A., Kondo, K., Damberg, C., Low, A., Motúapuaka, M., Freeman, M., ... Kansagara, D. (2017). The Effects of Pay-for-Performance Programs on Health, Health Care Use, and Processes of Care. Annals of Internal Medicine, 166(5), 341. Doi:10.7326/m16-1881 
Moller, A., Jager, A., Williams, G., y Kao, A. (2019). US Physicians' Work Motivation and Their Occupational Health. Medical Care, 57(5), 334-340. https://doi.org/10.1097/MLR.0000000000001101

Nguyen Duc Thanh, Phung Thanh Hung, Bui Thi My Anh, Tran Quynh Anh, Pham Quynh Anh \& Chu Huyen Xiem (2020) Developing a job motivation scale: A focus on cross-cultural adaptation and validation among hospital staff. International Journal of Healthcare Management,16. doi: 10.1080/20479700.2020.1752985

Pebes Mendoza, A., Uribe Rosas, C., y Loyola Aguije, G. (2019). Motivacion y su relacion con la satisfaccion laboral del profesional de enfermeria en el servicio de medicina del Hospital Regional de Ica 2016. Rev. enferm. Vanguard., 7(1), 13-22. https://doi.org/10.35563/revan.v7i1.18

Porter, T. H., Riesenmy, K. D., \& Fields, D. (2016). Work environment and employee motivation to lead: Moderating effects of personal characteristics. American Journal of Business, 31(2), 1-35. https://doi.org/10.1108/AJB-05-2015-0017.

Ramirez Motta, G. (2017). Calidad de vida del profesional de salud y motivación laboral en el servicio de emergencia del Hospital Nacional Hipólito Unánue, Lima.2016. [Tesis de Maestria, Universidad Cesar Vallejo]. Repositorio institucional. https://hdl.handle.net/20.500.12692/21830

Robbins, S., \& Coulter, M. (2014). Administracion (Decimo segunda ed.). Mexico: Pearson Educacion Mexico.

Ruiz-Fernandez, M., Ortega Galán, A., Fernandez Sola, C., Hernandez Padilla, J., Granero Molina, J., y Ramos Pichardo, J. (2020). Occupational Factors Associated with Health-Related Quality of Life in Nursing Professionals: A Multi-Centre Study. Int. J. Environ. Res. Public Health, 17(982), 2-12. doi:10.3390/ijerph17030982

Ryan, R. M. \& Deci, E. L. (2000a). Self-determination Theory and the facilitation of intrinsic motivation, social development, and well-being. American Psychologist, 55(1), 68-78. http://doi.org/10.1037/0003-066X.55.1.68.

Ryan, R. M. \& Deci, E. L. (2000b). Intrinsic and Extrinsic Motivations: Classic Definitions and New Directions. Contemporary Educational Psychology, 25, 5467. https://doi.org/10.1006/ceps.1999.1020. 
Ryan, R., \& Deci, E. (2020). Intrinsic and extrinsic motivation from a self-determination theory perspective: Definitions, theory, practices, and future directions. Contemporary Educational Psychology, 30(30), 1-11. https://doi.org/10.1016/j.cedpsych.2020.101860

Soto, M. (2018). Estudio de la Motivacion Laboral en el personal del Centro de Salud la Esperanza 2018. Revista Medica Hospital Hipolito Unanue, 11(2), 27-31. https://revista.hospitaltacna.gob.pe/index.php/revista2018/article/view/60

Studer, B. \& Knecht, S. (2016). Motivation: Theory, neurobiology and applications. ProQuest Ebook Central. https://ebookcentral.proquest.com

Varghese, S., \& Jayan, C. (2013b). Quality of work life: A dynamic multidimensional construct at work place- part II. Guru Journal of Behavioral and Social Science, 1(2), 91-104. http://gjbss.org/wp-content/uploads/2013/03/GJBSS-paper-V1-I25-susan-+-Jayan.pdf 